REVISTA ACADÉMICA ECO (2020) 22: 57-75

\title{
NOTAS BREVES SOBRE LA TEORÍA PROSPECTIVA Y SU APLICACIÓN EN EL CAMPO DE LA ECONOMÍA
}

Artículo de investigación científica

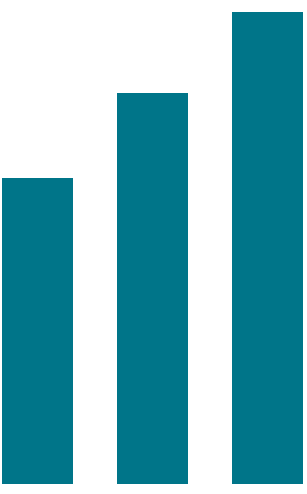

\section{Juan Fernando Díaz Lara}

Candidato a doctor en Economía Aplicada por la Universidad del País Vasco; Master of Science en Globalización y Desarrollo Económico por la Universidad de Amberes, Bélgica.

Correo: fernandoecon@gmail.com

Fecha de recepción: 14 de noviembre de 2019

Fecha de aceptación: 29 de noviembre de 2019

\section{Resumen}

El presente artículo estudia la aplicación de la teoría prospectiva a partir de las ideas propuestas por Kahneman y Tversky (2007) y Kahneman (2011), como un enfoque alternativo para la elección de opciones en el proceso de toma de decisiones dentro del campo de la economía conductual, y como una crítica al pensamiento convencional propuesto por la teoría de la utilidad esperada, enunciada por Bernuolli (1738) y desarrollada matemáticamente por Von Neumann \& Morgenstern (1944). Para ello, se presentan los planteamientos teóricos fundamentales de la teoría prospectiva en un lenguaje narrativo (no matemático), pretendiendo abarcar audiencias fuera del campo económico o estadístico, seguido de una exposición de casos aplicados bajo los parámetros de esta teoría innovadora y finalmente, se exponen los hallazgos encontrados como producto de un ejercicio aplicado a treinta estudiantes de la carrera de Economía Empresarial de la Universidad Rafael Landivar, con el fin de evaluar la elección en situaciones de decisión en campos de ganancias y pérdidas. Finalmente, las conclusiones apuntan a la necesidad, tanto de incluir la enseñanza de este tipo de herramientas innovadoras en los programas de carrera (no solo de Economía Empresarial), así como de profundizar en el desarrollo científico de esta rama de la economía conductual.

Palabras clave: economía conductual, teoría de prospectiva, economía, teoría de la utilidad esperada 


\title{
BRIEF NOTES ON THE PROSPECTIVE THEORY AND ITS APPLICATIONS IN THE FIELD OF ECONOMY
}

Scientific investigation article

\begin{abstract}
The present article considers the application of the prospective theory based on the ideas of Kahneman \& Tversky (2007) and Kahneman (2011) as an alternative focus on option selection in the decision-making processes of behavioral economics, and as a critique of conventional thinking in the theory of expected utility proposed by Bernuolli (1738) and developed mathematically by Von Neumann \& Morgenstern (1944). To this end, the theoretical approaches of prospective theory are described in a narrative and not mathematical language, in order to reach audiences outside the fields of Economy and Statistics, followed by the presentation of cases in which the parameters of this innovative theory were applied. Likewise, the article presents the results of an exercise performed on thirty Business students at University Rafael Landivar in order to evaluate decision making choices in profit and loss situations. The final conclusions highlight the importance of including this type of innovative tools in study programs (not only in Business programs), as well as of strengthening the scientific development of this branch of Behavioral Economics.
\end{abstract}

Key words: behavioral economics, prospective theory, economy, theory of expected utility 


\section{Introducción}

El análisis de la elección dentro del proceso de toma de decisiones por parte de los individuos se basa en los supuestos fundamentales de la teoría de la utilidad esperada, propuesta a mediados del sigloXVIl y formulada (con carácter neoclásico) a mediados del siglo $X X$.

A la fecha, gran parte de los análisis de la conducta del consumidor en el campo económico y principalmente dentro del campo de la economía como disciplina, siguen realizándose bajo este enfoque, asumiendo que el consumidor o más ampliamente, el tomador de decisiones, basa sus elecciones de acuerdo con una conducta racional, que se mantiene operando bajo el cumplimiento estricto de ciertos supuestos (llamados axiomas) y que mantienen así la validez de un modelo teórico que la evidencia parece refutar.

Este artículo presenta una revisión breve sobre la teoría prospectiva desarrollada por Kahneman y Tversky, como un enfoque que no necesariamente tuvo como punto de origen la teoría neoclásica y que aporta herramientas valiosas para mejorar la comprensión y el análisis en la toma de decisiones de las personas, lo que evidencia que existen ciertos procesos cerebrales y de contexto que afectan la toma de decisiones y que provocan que esta no necesariamente sea siempre racional. 


\section{Aspectos teóricos}

\subsection{Una paradoja que resulta en axiomas de elección}

La teoría prospectiva fue propuesta por Kahneman y Tversky (2007) para demostrar, a través de numerosos experimentos altamente controlados, que la mayor parte de las personas viola sistemáticamente los axiomas básicos de la teoría de la utilidad esperada en sus procesos de toma de decisiones.

Como referencia, debe considerarse que los planteamientos fundamentales de la teoría de la utilidad esperada fueron propuestos por Bernuolli (1738), al momento de resolver la llamada «Paradoja de San Petersburgo» (considerar las distintas publicaciones derivadas a partir del mismo Bernuolli y su análisis de decisiones en condiciones de riesgo, para conocer a detalle y profundizar sobre esta paradoja), un problema que ocupó el pensamiento de economistas y estadísticos durante el siglo XVIII, abordando una formulación teórica planteada en economía para representar el caso en donde, considerando únicamente el valor esperado como único criterio de decisión, el tomador de decisiones estará equivocado y elegirá una opción irracional.

El planteamiento fue resuelto por Bernuolli, a través de evidenciar las diferencias entre dos conceptos: el valor y la utilidad esperados, reforzando la idea de que esta última considera la utilidad en conjunto con las probabilidades (en términos estadísticos, esto conllevaría a asumir que estas probabilidades son multiplicativas a la utilidad y no ponderativas).

Para reforzar los aportes en este campo, Von Neumann \& Morgenstern (1944) desarrollaron la formulación matemática para sustentar la solución de Bernuolli, proponiendo un conjunto de condiciones (axiomas) inherentes a las relaciones preferenciales con el fin de asegurarse de que la función de utilidad esperada operara de forma correcta y los individuos evidenciaran así una conducta racional. En síntesis, los axiomas propuestos (y los más básicos) fueron los siguientes:

- Completud: la persona tiene un sistema de preferencias bien definidas, y asumiendo la existencia de las opciones $A$ y $B$, puede preferir $A$ a $B$, o bien, $B$ a $A$, o en todo caso, A y $B$ son indiferentes.

- Transitividad: las preferencias de la persona, además de cumplir con el axioma de completud, deben evidenciar consistencia. Por ejemplo, si se agrega una opción $\mathrm{C}$, de preferir $\mathrm{A}$ a $\mathrm{B}$ y $\mathrm{B}$ a $\mathrm{C}$, la persona preferirá con toda certeza A a C. 
- No saciedad: el individuo tenderá a preferir opciones que suponen una mayor cantidad de bienes sobre opciones que contengan un menor número de ellos y este comportamiento se mantendrá de forma indefinida (a excepción de que sean males, en donde la preferencia será a la inversa).

- Continuidad: si se consideran las tres opciones A, B y C, y existe una mayor preferencia de $\mathrm{A}$ a $\mathrm{B}$ y de $\mathrm{B}$ a $\mathrm{C}$, la persona podrá establecer un nivel de probabilidad para seguir indiferente entre $B$ y un conjunto de preferencias compuestas en donde A será elegida con una probabilidad «p» y C saldrá elegida con una probabilidad «1- p».

\subsection{Una alternativa a la teoría neoclásica}

De acuerdo con Kahneman \& Tversky (2007), la teoría neoclásica de la utilidad esperada no puede ser considerada necesariamente como el origen de la teoría prospectiva, sino más bien esta última debe asociarse a un enfoque independiente y alternativo para explicar la forma en que los individuos toman sus decisiones.

En general, los autores proporcionaron en esta teoría alternativa de elección, un marco de referencia respaldado empíricamente para describir con precisión cómo las personas realmente toman sus decisiones, prediciendo que los individuos tienden a ser reacios al riesgo en un dominio de ganancias, o cuando las cosas van bien, y que, por el contrario, buscan más riesgos (en términos relativos) en un dominio de pérdidas, como en situaciones en las cuales el tomador de decisiones puede estar atravesando una situación de crisis.

Como elemento innovador, la teoría prospectiva se basa en modelos psicofísicos, como los que originalmente inspiraron la propuesta de valor esperada de Bernoulli, aunque, debido al momento histórico, con menos herramientas sofisticadas a su alcance.

Debe considerarse por tanto que, tradicionalmente, la psicofísica investiga la relación precisa, generalmente expresada matemáticamente, entre los mundos físico y psicológico, con el objetivo de determinar el punto en el que el sujeto percibe psicológicamente un cambio en el estímulo físico como un cambio sensorial. Esto, considerando que la mayoría de las investigaciones en el dominio sensorial, por ejemplo, han determinado que el estímulo físico debe aumentar geométricamente para que la experiencia psicológica aumente aritméticamente (García et al., 1987).

Kahneman \& Tversky (2007) aplicaron estos principios psicofísicos para investigar el juicio y el proceso de toma de decisiones. Así como las personas no están conscientes del proceso cerebral que tiene lugar para trasladar la operación de la visión a la vista percibida sensorialmente, a la vez no se es consciente de las clases de cálculos que realiza el cerebro para evaluar una opción y con esto, las personas toman decisiones de acuerdo con la forma en que sus cerebros procesan 
y entienden la información y no solamente sobre la base de la utilidad esperada subjetiva que una determinada opción representa para un tomador de decisiones, desestimando así los axiomas de la elección racional (normativa).

Gran parte del trabajo de Kahneman \&Tversky (2007) está diseñado para demostrar que las teorías descriptivas y normativas no pueden combinarse en un único y adecuado modelo de decisión. En su lugar, las violaciones de comportamiento de la utilidad esperada en la cual las personas sistemáticamente se encasillan, son muy básicas para permitir la integración que los axiomas requieren para el funcionamiento de los modelos normativos.

En suma, las teorías que son empíricamente acertadas por modelos normativos fallan en cumplir incluso las prescripciones normativas más básicas. Por lo tanto, los autores en última instancia sostienen que las teorías normativas deben abandonarse por completo a la hora de analizar el juicio y el proceso de toma de decisiones porque no ofrecen una comprensión adecuada del comportamiento para la toma real de estas decisiones.

\subsection{El funcionamiento del nuevo enfoque}

La teoría prospectiva sugiere que es posible observar que la gente a menudo incumple los requerimientos más básicos de la teoría subjetiva de la utilidad esperada, sobre todo en proveer evaluaciones acertadas y confiables de la utilidad. Debido a que es muy difícil para las personas evaluar utilidades, el análisis se fundamenta en postulados mucho más fuertes que parten de la redefinición de los niveles de utilidad percibida con base ahora en la teoría psicológica y asociada a la experimentación.

De esta manera, la teoría prospectiva adelanta la noción de utilidad en una dirección útil y adecuada, incorporando la visión de que las curvas de utilidad difieren en una situación de ganancia de aquellas en una situación de pérdida. Más aún, la forma de las curvas de valor (otrora de utilidad) de la teoría prospectiva es similar para todos los individuos y como resultado, un analista no necesitaría conocer la utilidad particular de un tomador de decisiones individual en cada caso para formular inferencias, proyectar o diseñar estrategias.

Por lo tanto, mientras la teoría prospectiva puede aparentar ser analíticamente menos rigurosa y precisa que los modelos de utilidad esperada, es de hecho suficientemente precisa para permitir predicciones sobre la base de si una decisión tiene lugar en una situación de ganancias o pérdidas. De hecho, en materia analítica, la teoría prospectiva es más adaptable que la teoría subjetiva del modelo de utilidad esperada, ya que esta última podría requerir más información acerca de la curva de utilidad individual antes de que la predicción sea posible.

La teoría prospectiva está diseñada para explicar un patrón común en el proceso de toma de decisiones, siendo a la vez descriptiva y empírica en su naturaleza. Esta 
teoría se enfoca en dos partes del proceso de toma de decisiones: la fase de edición y la fase de evaluación. La primera engloba lo que ampliamente se conoce como los efectos de encuadre, incorporando el proceso de escoger dentro de ciertas opciones y de cómo esta decisión es influenciada por dos procesos subsecuentes: uno relacionado con el valor subjetivo, y el otro por la probabilidad percibida. A continuación, se explican con mayor detalle.

\subsubsection{Efectos de edición (o de encuadre)}

La edición o encuadre (llamado también «de marco») es la primera fase de la teoría prospectiva. Esta fase inicial se refiere a una representación de los actos, resultados y contingencias que están asociados con un problema particular a la hora de afrontar una elección. El encuadre incorpora un número de operaciones básicas que simplifica y provee el contexto de esta elección.

Los efectos de encuadre, considerados a partir de Kahneman (2011), se refieren por tanto a la forma en la cual una elección o una opción pueden verse afectadas por el orden o la manera en la cual se presentan a un tomador de decisiones, siendo un concepto crucial por varias razones. En primera instancia, un tomador de decisiones no sabe la totalidad de opciones relevantes que están disponibles, y debe escoger sobre la base de las más evidentes hasta ese momento. En cierta medida, esta actividad de determinar y figurar las opciones disponibles constituye el corazón del proceso creativo de la toma de decisiones.

Con lo anterior, la elección puede estar afectada por manipulaciones relativamente triviales en la consideración de las opciones disponibles y, por lo tanto, la mayor parte de los tomadores de decisión argumentarían que ciertas transformaciones aparentemente inocuas, como el orden en que se presentan las opciones, no deberían de afectar sustantivamente las decisiones de elección. La paradoja es que los efectos de encuadre a menudo están incrustados en los problemas de decisión de tal manera que pocos tomadores de decisión se dan cuenta del impacto desproporcionado que estos efectos de encuadre tienen sobre ellos.

Los tomadores de decisión con frecuencia desconocen estos efectos de encuadre y recurren a evaluaciones intuitivas de opciones predeterminadas porque a menudo les es imposible reconocer la forma en que se violan procedimientos más racionales en la determinación original de estas opciones preestablecidas. Por lo tanto, cualquier modelo de elección descriptivamente adecuado debe ser sensible a estos efectos de encuadre.

De acuerdo con Kahneman (2011), el encuadre está controlado por la manera en la cual el problema de la elección se presenta, así como por las normas, los hábitos y las expectativas del tomador de decisiones. Estas normas y hábitos pueden ser muy idiosincráticos y las expectativas pueden verse afectadas por varios sesgos cognitivos. Sin embargo, las operaciones de encuadre concernientes a la manera en la cual los problemas son presentados pueden describirse sistemáticamente. 
El propósito detrás de encuadrar varias opciones es simplificar la evaluación de elecciones que están disponibles para un tomador de decisiones. Este proceso es realizado a través del uso de varias clases de procedimientos, en donde el más importante tiene que ver con la segregación, pero también incluyendo mecanismos como codificaciones, combinaciones y cancelaciones.

\section{a. Los mecanismos del encuadre}

Dentro de los mecanismos de encuadre, la segregación corresponde a la idea de que cuando las personan realizan elecciones, tienden a enfocarse en los factores a la mano que aparentan ser más relevantes al problema inmediato que se les presenta. De hecho, los tomadores de decisión no tienden a darse cuenta de manera adecuada de los factores relacionados que pueden tener un impacto real en el resultado, pero que no parecen ser directamente relevantes para la elección específica en cuestión.

La codificación se refiere así a la tendencia de las personas a categorizar los resultados en términos de pérdidas y ganancias, en lugar de hacer una valoración absoluta con relación a la riqueza o bienestar, por ejemplo; mientras que la combinación es una estrategia de encuadre que se refiere a la tendencia de las personas a sumar de manera conjunta la probabilidad de las elecciones que presentan resultados idénticos.

Seguidamente, la cancelación se refiere al descuento involucrado en la evaluación de elecciones que llevan al mismo resultado. Por lo tanto, si una parte de una opción es la misma a lo largo de los conjuntos de elecciones, este aspecto tiende a ser ignorado en la evaluación de los prospectos.

Estos aspectos que constituyen el encuadre de un problema son importantes por la forma en que pueden afectar la elección. Por ejemplo, la codificación per se puede determinar el punto de referencia relevante y ayudar a definir los resultados, actos y contingencias asociadas con una elección. Concretamente, a través de incluir y excluir otras opciones, el proceso de encuadre crea las opciones que se entiende que están disponibles para un tomador de decisiones en un tiempo determinado.

El aspecto crucial de los efectos de encuadre tiene que ver con su carácter contranormativo, sobre todo con respecto a la teoría de la elección desde un enfoque neoclásico. La razón primordial es que los efectos de encuadre pueden provocar cambios en las preferencias que violan las teorías racionales, requiriendo axiomas de invarianza para operar. Con los efectos de encuadre, las personas tomarán elecciones distintas basadas solamente en el orden en que las opciones se presenten $y$, en suma, la misma decisión podrá generar diferentes elecciones dependiendo de cómo se presente la situación (este punto es especialmente contradictorio para cualquier teoría normativa de toma de decisiones). 
En todo caso, si a las personas se les mostrara que son influenciadas por los efectos de encuadre, estarían de acuerdo en que no son tan afectadas por este. Desde una perspectiva normativa del proceso de decisiones, estos elementos son perturbadores, debido a que las personas que encaran un problema de decisión y tienen una preferencia definida, pueden tener una preferencia diferente en un marco de referencia distinto para un mismo problema. En general, los individuos son normalmente inconscientes de los marcos de referencia alternativos y de sus potenciales efectos en el atractivo relativo de las opciones existentes, deseando que sus preferencias fueran independientes del marco de referencia, y evidenciando incertidumbre a la hora de resolver inconsistencias no detectadas.

\subsection{Valoración general}

La exposición teórica realizada hasta ahora pretende exponer los elementos básicos de la teoría prospectiva para explicar la elección en el proceso de toma de decisiones de las personas. Si bien el campo es innovador dentro de la teoría económica, el cuerpo teórico es extenso y sofisticado, sobre todo debido al carácter crítico de este enfoque con respecto a los postulados tradicionales de la toma decisiones (normalmente enunciados en la teoría del consumidor/teoría de la utilidad).

No obstante, más allá de los esfuerzos matemáticos y demostrativos para sustentar las críticas al enfoque neoclásico convencional, los argumentos teóricos presentados se han articulado con el ánimo de presentarlos en un lenguaje más narrativo que matemático, asumiendo que el conocimiento de esta vertiente es todavía incipiente y que el mismo puede beneficiar el alcance del análisis en el proceso de toma de decisiones para distintos agentes económicos en diversos sectores, incluyendo: análisis más sofisticados de la conducta del consumidor, la propensión o aversión al riesgo en opciones financieras, la formulación de modelos de rendimientos y retornos ante situaciones de riesgo específicas y la gestión de estrategias para mejorar la penetración en mercados particulares, entre otras.

En todo caso, los postulados teóricos seleccionados para este apartado pretenden brindar una base adecuada para la aplicación del ejercicio planteado en este documento y que corresponde a la evaluación de las opciones ante situaciones de riesgo en estados de pérdidas y ganancias de manera simulada. 


\section{Aplicación de la teoría prospectiva}

En este apartado, se expone una reseña de tres estudios para ilustrar la aplicabilidad de la teoría prospectiva en distintos campos, no necesariamente relacionados. Dentro de estos estudios, se incluye uno desarrollado en Colombia y otro en España, considerando que, a la fecha, el campo de estudio científicamente formalizado para esta teoría es todavía incipiente en Guatemala.

\subsection{Teoría prospectiva y consumo de tabaco en Colombia}

Monroy-Cely (2011) hizo referencia a las cifras de la Organización Mundial de la Salud (OMS), que para 2010 estimaron que el consumo de tabaco en el mundo provocó la muerte de cerca de cinco millones de personas por año, equivalentes a una relación de uno de cada diez adultos en el mundo.

Desde el punto de vista económico, esta relación es dramática si se toma en cuenta que el incremento del gasto público en materia de salud y la reducción en los niveles de productividad del factor trabajo, condicionan las posibilidades de mejorar las condiciones de desarrollo económico de los países.

Para el caso colombiano, el Instituto Nacional de Cancerología de ese país indicó que ocho de cada diez consumidores de tabaco iniciaban a fumar antes de los dieciocho años y cerca de tres de cada diez adolescentes en 2010 eran fumadores; ello provocaba un mayor panorama de riesgo en el padecimiento de enfermedades respiratorias y padecimientos de mayor gravedad como el cáncer.

Con el fin de abordar la problemática desde un enfoque estratégico, MonroyCely (2011) aplicó elementos de la economía conductual relacionados con la teoría prospectiva y su efecto de marco, al campo del derecho para analizar el efecto de las regulaciones vinculadas con la intención de disuadir el consumo de tabaco en Colombia.

Para realizar el estudio, el autor analizó el efecto de los mensajes de advertencia impresos en las cajetillas de cigarros, concluyendo que este tipo de mensajes sí tiene posibilidades de reducir eficazmente la incidencia en el consumo de tabaco, pero de una forma condicionada, evidenciando que una mayor efectividad podría estar correlacionada con formas alternativas de marcaje en las cajetillas (formas más gráficas), distintas a las tradicionales que solo se refieren a redactar la consecuencia negativa de fumar que apela a la racionalidad de evitarlo. 


\subsection{Teoría prospectiva y compra de vivienda en España}

García-Badell (2014) se planteó estudiar los llamados efectos de anclaje y ajuste, de aislamiento y de prominencia, y su impacto en las decisiones de compra de vivienda en España.

Para realizar el estudio, el autor consideró tres hipótesis: i) que en general, la valoración que los compradores realizan de los atributos cualitativos de la vivienda dependen de la valoración hecha de esos atributos al momento de evaluar la primera vivienda; ii) que en orden de simplificar su elección, a las personas les es indiferente el conjunto de atributos cualitativos que son comunes a las otras alternativas y se enfocan en los atributos que hacen que una opción se distinga más sobre las otras; y iii) que en general, al momento de evaluar los atributos cualitativos de una vivienda, el consumidor establece relaciones entre estos atributos, permitiéndole comparar los atributos entre ellos y finalmente, permitiéndole comparar las alternativas de compra.

Dentro de sus conclusiones, García-Badell (2014) planteó que el efecto de anclaje y de ajuste no solo afecta el precio de los atributos, sino que además los cualifica. Asimismo, que con base en los resultados obtenidos, los aspectos positivos de un producto parecen estar más anclados que los aspectos negativos, y que la señal del ajuste no siempre es negativa.

De igual forma, el autor concluyó que la decisión de compra tiende a simularse mediante un problema de aparejamiento: los participantes tienen que escoger luego de evaluar diferentes aspectos de cada casa. No obstante, de acuerdo con el efecto de prominencia y sus consecuencias para el aparejamiento de las decisiones, los participantes establecen relaciones dentro de los atributos cualitativos que les permiten comparar cada alternativa en un mismo rango de utilidad.

Los resultados finalmente muestran que las personas tienden a anclar aspectos positivos para la primera opción de compra; asimismo, que los vendedores evitan mostrar como primera opción una casa que es prominente en solo un atributo específico y que, en todo caso, los vendedores deberían evitar enfocarse en un único atributo que se evidenció en la primera visita, provocando así que los consumidores comparen distintas características con el fin de transformar una actividad de elección en una de aparejamiento.

\subsection{La evidencia}

\subsubsection{Un ejercicio de aplicación}

Para ilustrar la aplicabilidad de la teoría prospectiva en el campo económico, se consideró la realización de un ejercicio como sondeo para evaluar su funcionamiento con alumnos seleccionados de la carrera de Economía Empresarial de la Facultad de Ciencias Económicas y Empresariales de la Universidad Rafael Landívar. 
Para fines de este estudio, no se priorizó la realización de un ejercicio estadístico controlado o riguroso en función de validez y representatividad, sino más bien un sondeo no probabilístico con treinta alumnos de la jornada vespertina, en los cursos de Fundamentos de Microeconomía y Tesis I, ubicados en el rango de edad de los 19 a los 22 años y de los cuales ocho fueron mujeres.

La intención de realizar este ejercicio consideró los siguientes propósitos:

- Demostrar la accesibilidad de la innovación en la generación de conocimiento: al considerar que no solo la Facultad de Ciencias Económicas y Empresariales sino la universidad como un todo, cuenta con una población cautiva y ávida para desarrollar estudios experimentales que pueden contribuir a generar conocimiento y ser pioneros en el desarrollo de la teoría prospectiva y otros campos relacionados.

- Desmitificar el método de la teoría prospectiva: que, dentro del campo de la economía conductual, se tiende a asociar la realización de experimentos con métodos muy sofisticados e inaccesibles, así como costosos y demandantes de equipos de medición sensorial que escapan al alcance del investigador.

- Evidenciar las elecciones en el proceso de toma de decisiones: ello forma parte de las interrogantes comunes de los estudiantes de Ciencias Económicas, que muestran recurrentemente interés por la economía conductual y que cuestionan los axiomas de la teoría neoclásica (impartidos en el curso de Fundamentos de Microeconomía que antecede al curso de Tesis I) como única explicación para estudiar la conducta del consumidor.

Para llevar a cabo el ejercicio, se delimitó la aplicación de la teoría prospectiva al análisis de decisión en situaciones simuladas de escenarios de pérdidas y ganancias, utilizando la plataforma electrónica SurveyMonkey, y diseñando un instrumento que, como encuesta con opciones de respuesta de marcaje, abarcó los siguientes planteamientos (la transcripción se realizó a partir de la encuesta original):

- Considera los siguientes escenarios y escoge qué prefieres (solo puedes marcar una opción):

- Tener la seguridad de ganar $\$ 4500$

- Probar suerte y ganar $\$ 10000$ o no ganar nada (50\% de probabilidad de cada opción)

- Considera los siguientes escenarios y escoge qué prefieres (solo puedes marcar una opción):

- Resignarse a perder $\$ 4500$

- Probar suerte y salvarse de perder dinero o perder $\$ 10000$ (50\% de probabilidad de cada opción) 
- Considera los siguientes escenarios y escoge qué prefieres (solo puedes marcar una opción):

- Ganar \$1000 con seguridad

- Probar suerte y no ganar nada o ganar $\$ 1500$ con un $90 \%$ de probabilidades

- Considera los siguientes escenarios y escoge qué prefieres (solo puedes marcar una opción):

- Resignarse a perder $\$ 1000$

- Probar suerte y no perder nada o perder \$1500 con un $90 \%$ de probabilidades.

- Asume que cuentas con un capital de \$1000. ¿Qué escoges? (solo puedes marcar una opción):

- Recibir \$500 más con seguridad

- Probarsuerte entre no recibirnada o ganar $\$ 1000$ (50\% de probabilidades)

- Asume que cuentas con un capital de \$2000. ¿Qué escoges? (solo puedes marcar una opción):

- Resignarse a perder $\$ 500$

- Probar suerte entre no perder nada o perder $\$ 1000$ (50\% de probabilidades)

\subsubsection{Presentación y discusión de los resultados}

De acuerdo con Kahneman (2011), la mayoría de las personas escogen la primera opción en el primer planteamiento, siendo esto coherente con la idea de que las personas tienden a ser aversas al riesgo cuando se encuentran en un dominio de ganancias. Por lo tanto, los alumnos deberían aceptar $\$ 4500$ seguros, aunque el valor esperado de la otra opción (la adición de la probabilidad de cada ganancia multiplicada por su valor) ascienda a $\$ 5000$. La respuesta obtenida se ilustra en la siguiente Figura 1. 


\section{Figura 1. Resultados a partir de la primera pregunta de análisis}

Considera los siguientes escenarios y escoge qué prefieres

(solo puedes marcar una opción)

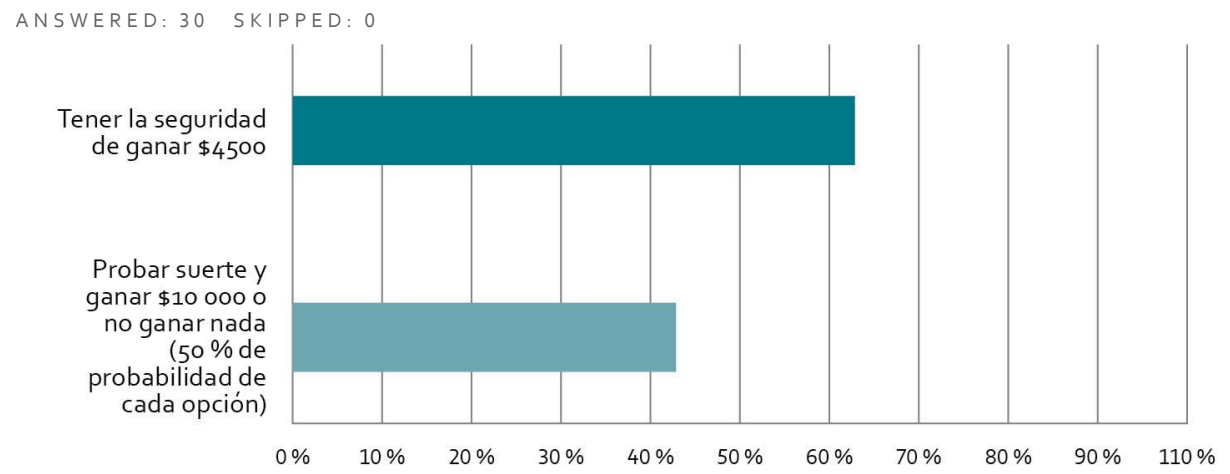

Fuente: elaboración propia con base en el ejercicio de aplicación.

En congruencia con lo sugerido a partir de la teoría prospectiva, la mayor parte de alumnos encuestados (73.33 \%) escogió un escenario de aversión al riesgo en un dominio de ganancias, a diferencia del $26.67 \%$ restante, que estuvo de acuerdo en arriesgar la ganancia simulada para obtener más ingreso.

Con respecto al segundo planteamiento, los resultados fueron los siguientes y se muestran en la Figura 2.

\section{Figura 2. Resultados a partir de la segunda pregunta de análisis}

Considera los siguientes escenarios y escoge qué prefieres (solo puedes marcar una opción)

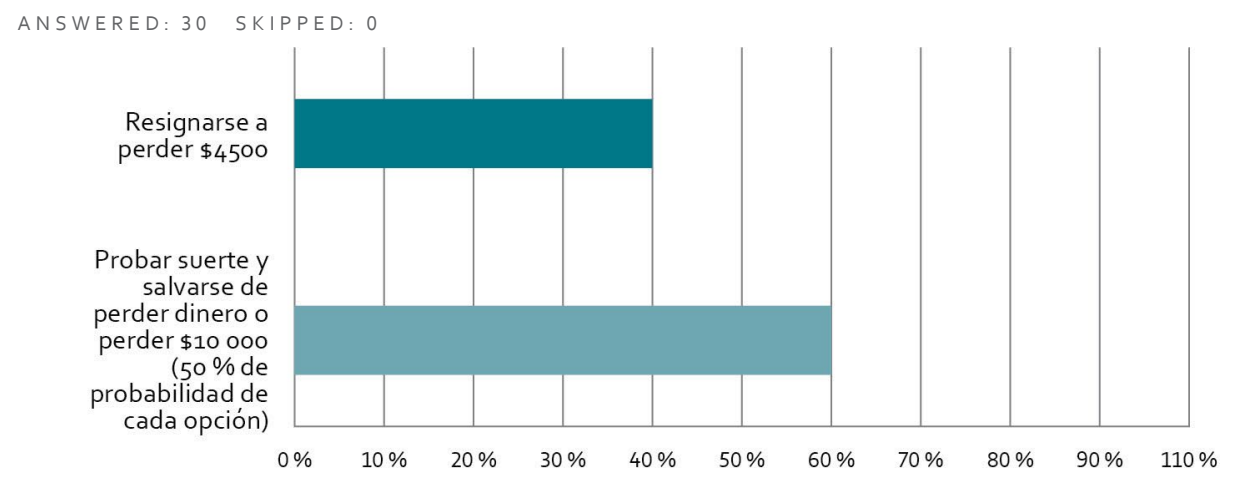

Fuente: elaboración propia con base en el ejercicio de aplicación.

Nuevamente, de acuerdo con lo sugerido por la teoría prospectiva, la respuesta de los estudiantes encuestados coincide con las predicciones del escenario, en donde se estima que las personas son propensas al riesgo en un dominio de pérdidas, apelando a la posibilidad de no incurrir en estas a diferencia de resignarse a tener una pérdida segura (aún con un monto menor fuera de las probabilidades). 
A partir de esta primera serie de planteamientos, puede inferirse que los estudiantes serían más prudentes a la hora de ganar y más arriesgados cuando existe la posibilidad de perder (6o \% estuvo en disposición de arriesgarse). Esto contrasta con las condiciones básicas de los axiomas de la teoría de la utilidad esperada en donde los individuos elegirían tanto la opción adversa al riesgo y segura de ganar $\$ 4500$ o resignarse a perder un monto similar; indiferentes al riesgo, probando suerte a ganar más o resignándose a perder, propensos al riesgo y arriesgándose por consiguiente en los dos escenarios que suponen esta condición en el ejercicio.

Si se considera un enfoque alternativo y complementario al ejercicio de referencia, se procede a mostrar ahora los resultados obtenidos para el tercer y cuarto planteamiento, considerándose para ello la Figura 3 y la Figura 4.

\section{Figura 3. Resultados a partir de la tercera pregunta de análisis}

Considera los siguientes escenarios y escoge qué prefieres (solo puedes marcar una opción)

ANSWERED: 30 SKIPPED: 0

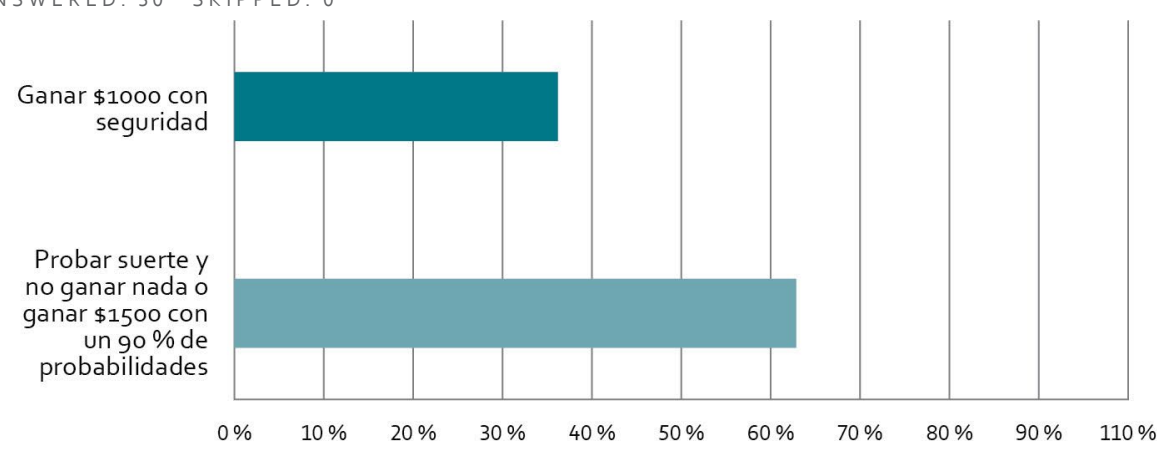

Fuente: elaboración propia con base en el ejercicio de aplicación.

\section{Figura 4. Resultados a partir de la cuarta pregunta de análisis}

Considera los siguientes escenarios y escoge qué prefieres (solo puedes marcar una opción)

ANSWERED: 30 SKIPPED: 0

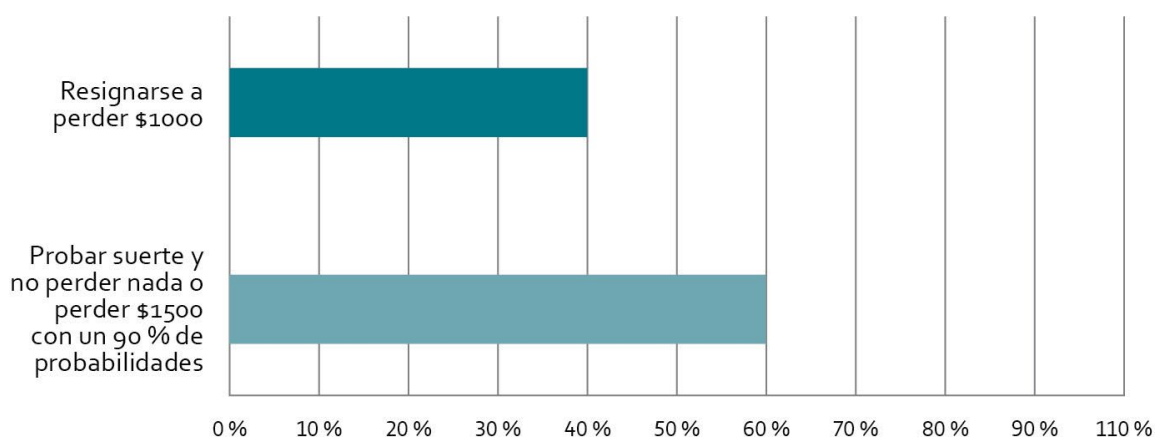

Fuente: elaboración propia con base en el ejercicio de aplicación. 
En este caso, las respuestas de los estudiantes nuevamente coinciden con el planteamiento de la teoría prospectiva. En la primera pregunta, se consideraba que la mayor parte de las personas sería aversa al riesgo, debido a que el valor subjetivo de $\$ 4500$ es mayor que el $90 \%$ de una ganancia de $\$ 1000$.

En este caso (tercera pregunta), por el contrario, la mayor parte de los estudiantes ha decidido arriesgarse (60\%) debido a la «ilusión» de la explicación que señala que la aversión al riesgo desde un enfoque de probabilidades es menos severa que la mención explícita de una pérdida absoluta que, a la luz del tomador de decisiones, se asocia con una pérdida mayor, siendo exactamente la misma.

Considérese ahora un escenario de control, en donde las dos últimas preguntas presentan un escenario similar de riesgo, pero en donde el planteamiento y el enfoque de la cuestión puede incidir en la toma de decisiones. Véase para ello las Figuras 5 y 6.

\section{Figura 5. Resultados a partir de la quinta pregunta de análisis}

Asume que cuentas con un capital de $\$ 1000$ ¿Qué escoges?

(solo puedes marcar una opción)

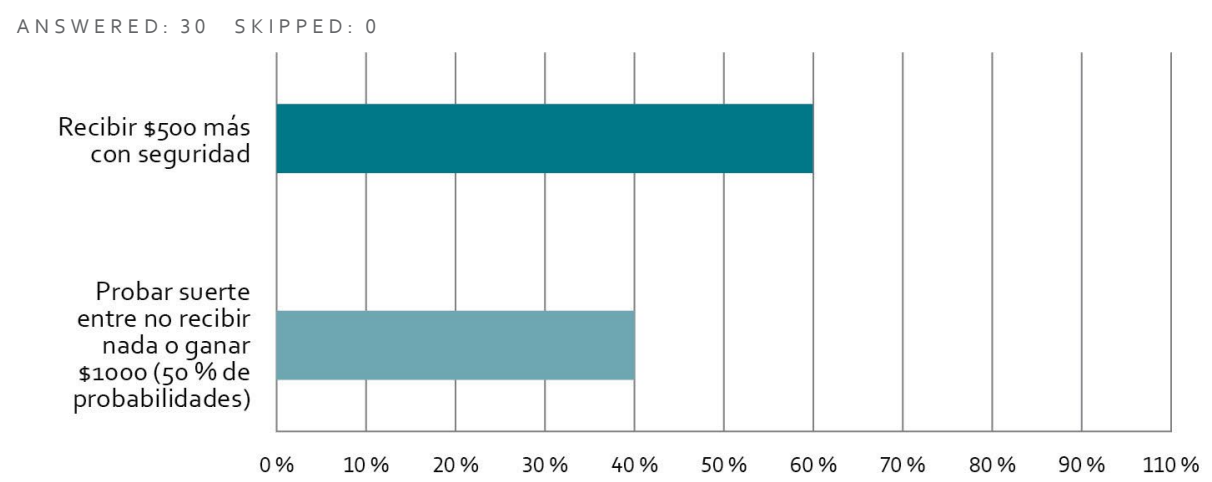

Fuente: elaboración propia con base en el ejercicio de aplicación. 


\section{Figura 6. Resultados a partir de la sexta pregunta de análisis}

Asume que cuentas con un capital de $\$ 2000$ ¿Qué escoges?

(solo puedes marcar una opción)

ANSWERED: 30 SKIPPED: 0

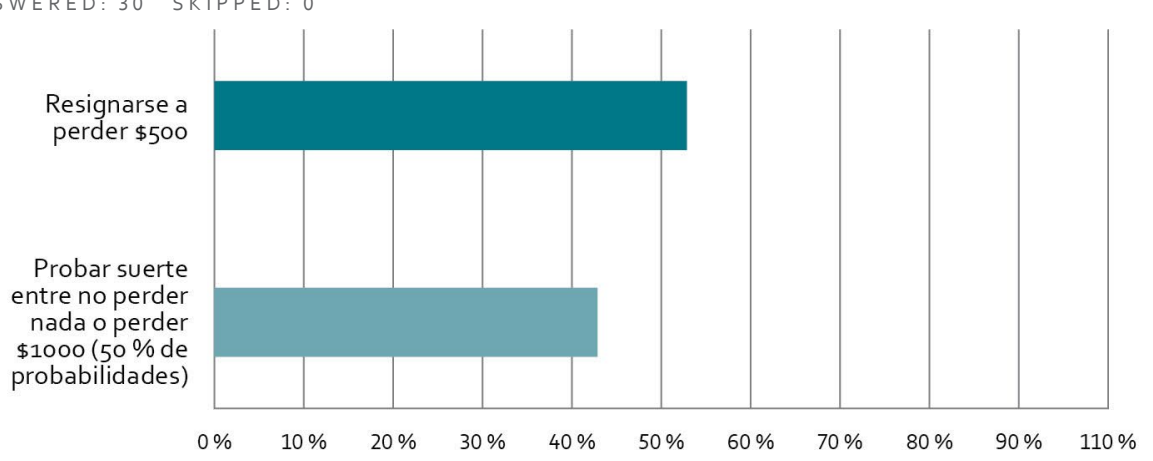

Fuente: elaboración propia con base en el ejercicio de aplicación.

De acuerdo con las especificaciones del ejercicio y a partir del diseño de evaluación de la teoría prospectiva, los escenarios planteados en las preguntas 5 y 6 son idénticos bajo el supuesto de que lo único que importa es el ingreso final que las personas tengan, como se asume en la teoría de la utilidad esperada.

Contrario a la mayor parte de experimentos citados por Kahneman (2011), los resultados de los estudiantes son congruentes con el enfoque neoclásico a pesar de que, en la primera decisión a considerar, el punto de referencia sea superior al monto considerado en la suma original y a que en la decisión dos, se pueda asumir una pérdida. La coincidencia entre las tasas de respuesta para cada pregunta evidencia de alguna manera la percepción idéntica por parte de los estudiantes encuestados. 


\section{Conclusiones}

El conocimiento y la aplicabilidad de la teoría prospectiva actualmente son todavía incipientes en el campo académico de Guatemala. Más allá de los esfuerzos de algunas instituciones educativas y de investigación de mercados para evaluar casos de economía experimental, la economía conductual como tal no ha sido desarrollada ni incorporada como un campo de estudio en la mayoría de los programas de carrera que podrían beneficiarse con esta disciplina.

En general, la economía conductual tiende a asociarse con un planteamiento científico menos riguroso y más costoso en la práctica que otros enfoques que fueron desarrollados a partir de la teoría neoclásica en economía, y que contribuyó a la formulación de la teoría de la utilidad esperada, como el principal (y único) eje de análisis para explicar la toma de decisiones de los agentes económicos. Si bien este enfoque es científicamente valioso, no debe ser visto como único y excluyente de otros alternativos que pueden facilitar una mejor comprensión del proceso de toma de decisiones y un análisis más certero, como el que plantea la teoría prospectiva.

Finalmente, la aplicación de la teoría prospectiva tiene el potencial de convertirse en un campo de análisis en el que la Universidad Rafael Landívar puede ser pionera, aprovechando la convergencia de elementos básicos para el análisis, como: una población cautiva y ad hoc para el desarrollo de cierto tipo de ejercicios de este corte; la existencia de carreras y programas que pueden beneficiarse con una mejor comprensión de la forma en que los agentes económicos eligen y dinamizan la actividad económica; y mediante la actualización e incorporación de otros enfoques más recientes en los programas de estudio para mantener la sintonía con el avance del pensamiento científico. 


\section{Referencias}

Bernuolli, D. (1738). Evolution and economics under risk. Recuperado de https://www. researchgate.net/publication/12302048_Daniel_Bernoulli_1738_Evolution_ and_economics_under_risk

García-Badell, G. (2014). Aplicación de la teoría prospectiva a la compra de vivienda. Recuperado de https://eprints.ucm.es/29642/1/T35978.pdf

García, R., Cronetto, J. y Rodríguez, M. (1987). Un enfoque psicofísico del sabor: aportes de la psicofísica a la tecnología de alimentos. https://www.redalyc.org/ pdf/805/80519311.pdf

Kahneman, D. y Tversky, A. (2007). Teoría Prospectiva: un análisis de la decisión bajo riesgo. Recuperado de https://catedradatos.com.ar/media/kahneman_ pensar.pdf

Kahneman, D. (2011). Pensar rápido, pensar despacio. Recuperado de https:// catedradatos.com.ar/media/kahneman_pensar.pdf

Monroy-Cely, A. (2011). Teoría prospectiva, efecto marco y los mensajes de disuasión de consumo de tabaco en Colombia. Recuperado de http://www.scielo.org.co/ pdf/vniv/n123/n123ao2.pdf

Von Neumann, J. y Morgenstern, O. (1994). Teoría de Juegos y Comportamiento Económico. Princeton: Princeton University Press. 